\title{
Measurement and correction of on- and off-momentum beta functions at KEKB
}

\author{
Akio Morita, Haruyo Koiso, Yukiyoshi Ohnishi, and Katsunobu Oide \\ High Energy Accelerator Research Organization, KEK, 1-1 Oho, Tsukuba, Ibaraki 305-0801, Japan
}

(Received 20 April 2007; published 27 July 2007)

\begin{abstract}
Optics correction is an important issue in the KEKB B-factory. Especially, the correction of beta functions is performed so as to reach near half-integer resonance of the horizontal betatron tune as closely as possible. Consequently, the luminosity can be improved during experiments of the B-meson in $e^{+} e^{-}$ collisions. Therefore, we have developed a correction method of the beta function not only at the designed beam energy, but also at an energy deviated from the nominal value. We present the procedures used to measure the behavior of the beta function, which depends on the momentum deviation, and the results compared with the model optics in the KEKB rings.
\end{abstract}

DOI: 10.1103/PhysRevSTAB.10.072801

PACS numbers: $41.85 .-\mathrm{p}$

\section{INTRODUCTION}

The KEKB B-factory is an energy-asymmetric doublering collider consisting of an $8 \mathrm{GeV}$ high-energy electron ring (HER) and a $3.5 \mathrm{GeV}$ low-energy positron ring (LER) [1]. In order to achieve a very high luminosity for mass production of B-mesons, KEKB was designed as a lowemittance lattice with a low beta interaction point. To obtain a large dynamic aperture for a long beam lifetime and good injection efficiency, a $2.5 \pi$ arc cell structure with noninterleaved sextupoles for chromaticity correction was introduced into the KEKB lattice design. The horizontal betatron tunes of the KEKB rings are set close to the halfinteger resonance as much as possible to achieve a higher luminosity [2]. The typical working tunes are (45.505, 43.534) for LER and $(44.509,41.565)$ for HER. KEKB has operated physics runs since 1999, and accumulated an integrated luminosity of $710 \mathrm{fb}^{-1}$ by December, 2006. A peak luminosity of $1.7118 \times 10^{34} \mathrm{~cm}^{-2} \mathrm{~s}^{-1}$ was achieved in November 15, 2006. The major machine parameters of the latest operation are given in Table I.

We conduct an 8-hour shutdown for maintenance every two weeks, and perform optics corrections before starting a physics run. The optics corrections, such as $x-y$ coupling, dispersions, and beta functions, usually take place at the designed beam energy (on-momentum) [2,3]. As a result of a beta correction at the on-momentum, the rms of the deviations from the model beta functions typically becomes within $5 \%$, and the betatron tunes are consistent within $10^{-4}$, respectively. The on-momentum beta correction has been successfully working and contributes to stable operation near to the half-integer resonance.

On the other hand, a disagreement in the betatron tune chromaticity between the model optics and the real machine has been observed. We sometimes found that the beam lifetime was shorter in the real machine than we had expected, although we optimized the strength of the sextupole magnets to make the dynamic aperture large enough in the model optics. This implies that the momentum dependence of the real optics is different from the model optics. In order to understand the disagreement, we measured the beta function by changing the rf frequency of the ring, and tried to correct the momentum dependence of the beta functions.

In the following sections, we describe in detail the measurement method and correction techniques for both the on-momentum and off-momentum optics.

\section{ON-MOMENTUM BETA MEASUREMENT AND CORRECTION}

\section{A. Beta measurement}

There are three kinds of typical measurement methods. The first method is the direct measurement of the free betatron oscillation by using the turn-by-turn beam position monitors (BPMs) [4]. The second method is the measurement of the betatron tune shift perturbed by changing the gradient of the quadrupole field [5]. The third method is the analysis of the closed-orbit responses perturbed by changing the dipole kicks [6,7]. It depends on both the controllable beam line elements and the available beam measurement devices which kind of measurement method is usable. In our KEKB rings, the measurement method using the turn-by-turn BPMs is not usable because of the lack of the number of the turn-by-turn BPMs. The method measuring the betatron tune shift by changing the field gradient is possible by using the individual subexcitation coil of the quadrupole magnets dedicated for the beambased-alignment, but it is too slow to measure the beta functions around the whole ring in the optics correction procedure before the luminosity run. Therefore, we choose the measurement method based on the analysis of the closed-orbit response perturbed by the dipole kicks.

In our beta measurement method, we measure a set of the closed-orbit responses to steering dipole kicks in the horizontal $(x)$ and vertical $(y)$ planes. By fitting the measured closed-orbit response with the linear perturbation model of the closed-orbit, both the beta function and the betatron phase advance are estimated. 
TABLE I. KEKB machine parameters on November 15, 2006.

\begin{tabular}{|c|c|c|c|}
\hline Parameter & LER & HER & Unit \\
\hline rf frequency & \multicolumn{2}{|c|}{508.88} & $\mathrm{MHz}$ \\
\hline Circumference & \multicolumn{2}{|c|}{3016} & $\mathrm{~m}$ \\
\hline Crossing angle at IP & \multicolumn{2}{|c|}{22} & $\operatorname{mrad}$ \\
\hline Number of bunches & \multicolumn{2}{|c|}{1388} & \\
\hline Beam current & 1662 & 1340 & $\mathrm{~mA}$ \\
\hline Horizontal emittance & 18 & 24 & $\mathrm{~nm}$ \\
\hline Betatron tunes $\left(\nu_{x} / \nu_{v}\right)$ & $45.505 / 43.534$ & $44.509 / 41.565$ & \\
\hline Beta functions at IP $\left(\beta_{x}^{*} / \beta_{y}^{*}\right)$ & $590 / 6.5$ & $560 / 5.9$ & $\mathrm{~mm}$ \\
\hline Beam-beam parameters $\left(\xi_{x} / \xi_{y}\right)$ & $0.117 / 0.105$ & $0.070 / 0.056$ & \\
\hline Peak luminosity (Belle CSI) & \multicolumn{2}{|c|}{1.7118} & $10^{34} \mathrm{~cm}^{-2} \mathrm{~s}^{-1}$ \\
\hline
\end{tabular}

The first-order perturbation of the closed-orbit response [8] is described as

$$
\begin{aligned}
\Delta \chi(s)= & \frac{\sqrt{\beta_{\chi}(s)}}{2 \sin \pi \nu_{\chi}} \Delta \theta_{\chi} \sqrt{\beta_{\chi}\left(s_{\text {kick }}\right)} \\
& \times \cos \left[\left|\phi_{\chi}(s)-\phi_{\chi}\left(s_{\text {kick }}\right)\right|-\pi \nu_{\chi}\right],
\end{aligned}
$$

where $\chi$ represents either $x$ or $y$, and $\triangle \chi(s), \beta_{\chi}(s), \phi_{\chi}(s)$, $\nu_{\chi}, \triangle \theta_{\chi}$, and $s_{\text {kick }}$ are the displacement of the orbit, the beta function, the betatron phase advance, the betatron tune, the kick-angle of the steering dipole magnet along the ring, and the location of the steering dipole magnet along the ring, respectively. Assuming that the steering dipole magnet does not overlap with the BPMs, the displacement of the closed orbit at the $i$ th BPM induced by the $j$ th steering dipole magnet is obtained by

$$
\begin{aligned}
\Delta \chi_{i}^{j}= & \frac{\triangle \theta_{j}}{2 \sin \pi \nu} \sqrt{\beta_{i}^{M}} \sqrt{\beta_{j}^{S}} \\
& \times \cos \left[\operatorname{sign}\left(\phi_{i}^{M}-\phi_{j}^{S}\right)\left(\phi_{i}^{M}-\phi_{j}^{S}\right)-\pi \nu\right],
\end{aligned}
$$

where superscripts ${ }^{M}$ and ${ }^{S}$ indicate the BPM and the steering dipole magnet, respectively. $\triangle \theta_{j}$ is the kick angle of the $j$ th steering dipole magnet.

In order to obtain the beta function, we fit Eq. (2) to the measured sets of the closed-orbit response by using the least-squares method. From the relationship between the number of unknown quantities and the number of equations, more than three kinds of the closed-orbit responses are required to fit the beta function. The betatron tune and the displacement of the closed-orbit are independently measured by a tune spectroscope and BPMs. The $\operatorname{sign}\left(\phi_{i}^{M}-\phi_{j}^{S}\right)$ term is given by the order of the beam line elements because of the monotonicity of the betatron phase advance.

For an optics correction, the beta function at the dipole magnet is not required. The product between the square of the kick angle of the dipole magnet and the beta function at the dipole magnet can be treated as a scaled beta function. Therefore, the accuracy of the kick angle could be neglected by discarding the beta function at the dipole mag- net. The remaining unknown variables in Eq. (2) are the beta function and the betatron phase advance at the dipole kicks and the BPMs. Alternatively, Eq. (2) is rewritten as follows:

$$
\begin{aligned}
\triangle \chi_{i}^{j}= & \left(\cos \pi \nu f_{j} X_{j}^{S}-S_{i j} \sin \pi \nu f_{j} Y_{j}^{S}\right) X_{i}^{M} \\
& +\left(\cos \pi \nu f_{j} Y_{j}^{S}+S_{i j} \sin \pi \nu f_{j} X_{j}^{S}\right) Y_{i}^{M} \\
= & \left(\cos \pi \nu X_{i}^{M}+S_{i j} \sin \pi \nu Y_{i}^{M}\right) f_{j} X_{j}^{S} \\
& +\left(\cos \pi \nu Y_{i}^{M}-S_{i j} \sin \pi \nu X_{i}^{M}\right) f_{j} Y_{j}^{S},
\end{aligned}
$$

where

$$
\begin{gathered}
f_{j} \equiv \frac{\triangle \theta_{j}}{2 \sin \pi \nu}, \quad S_{i j} \equiv \operatorname{sign}\left(\phi_{i}^{M}-\phi_{j}^{S}\right), \\
X_{i}^{l} \equiv \sqrt{\beta_{i}^{l}} \cos \phi_{i}^{l}, \quad Y_{i}^{l} \equiv \sqrt{\beta_{i}^{l}} \sin \phi_{i}^{l} \quad(l=M, S) .
\end{gathered}
$$

By denoting the measured displacement of the closed orbit as $\triangle \tilde{\chi}_{i}^{j}$, the square sum of the residual errors of the closedorbit responses is written by

$$
e^{2}=\sum_{i, j}\left(\triangle \tilde{\chi}_{i}^{j}-\triangle \chi_{i}^{j}\right)^{2}
$$

Applying either Eq. (3) or Eq. (4) to Eq. (6), we obtain a polynomial function of degree four. However, either for given $\left\{X_{i}^{M}, Y_{i}^{M}\right\}$ or $\left\{X_{j}^{S}, Y_{j}^{S}\right\}$, Eq. (6) becomes quadratic to $\left\{X_{j}^{S}, Y_{j}^{S}\right\}$ or $\left\{X_{i}^{M}, Y_{i}^{M}\right\}$, respectively. A unique solution of the least-squares fit to minimize the quadratic sums is obtained by using singular value decomposition (SVD).

In the fitting procedure, we use a brute-force approach, which improves the residual error by interleaving two types of the least-squares fit, as follows. At the first step, an initial value of $\left\{X_{i}^{M}, Y_{i}^{M}\right\}$ is given by the model optics, whose tunes have been adjusted to the measured betatron tunes. (The betatron tunes of the model optics are adjusted to be the measured tunes in advance.) In the second step, the new $\left\{X_{j}^{\prime S}, Y_{j}^{\prime S}\right\}$ is calculated from the given $\left\{X_{i}^{M}, Y_{i}^{M}\right\}$ by a least-squares fit of Eq. (4). In the third step, $\left\{X_{i}^{\prime M}, Y_{i}^{\prime M}\right\}$ is 
replaced by the least-squares fit of Eq. (3) using the obtained $\left\{X_{j}^{\prime S}, Y_{j}^{\prime S}\right\}$. Both the second and third steps are iterated until the square sum of the residual error, Eq. (6), has converged. This iteration method should converge because of a monotonic decreasing of the square sum of the residual error. The number of iterations sufficient to converge the relative improvement of the residual error $-\left(e_{n+1}^{2}-\right.$ $\left.e_{n}^{2}\right) / e_{n}^{2}$ within $10^{-8}$ is about $10^{4}$. The brute-force fitting requires about 5 minutes per plane in our computing environment.

However, the brute-force fitting scheme is too slow for the online optics measurement system. In order to improve the convergence speed, a general minimizing algorithm for a function of many variables is applied. The number of the BPMs is larger than the number of different kinds of measured closed-orbit distortions. KEKB has about 450 BPMs in each ring. On the other hand, we measure only six single-kicked orbits per plane. In order to reduce the cost of the minimizing algorithm, we chose $\left\{X_{j}^{S}, Y_{j}^{S}\right\}$ as the independent variable and $\left\{X_{i}^{M}, Y_{i}^{M}\right\}$ as the slave variable determined from $\left\{X_{j}^{S}, Y_{j}^{S}\right\}$ in the least-squares method. As the minimizing algorithm, we apply the conjugate gradient method of Polak-Rihiére (CGPR) [9] to the error function of $\left\{X_{j}^{S}, Y_{j}^{S}\right\}$ by using a numerical derivative. From the result of the CGPR method, $\left\{X_{i}^{M}, Y_{i}^{M}\right\}$ is obtained by the leastsquares method for Eq. (6) using Eq. (3). By using the CGPR method, the number of iterations is decreased to within 20 iterations, and the fitting is usually completed within 30 seconds. This CGPR method is sufficiently faster than the previous brute-force method and robust enough for a local minimum search.

In general, a more efficient algorithm could be given by using knowledge about the $\left\{X_{j}^{S}, Y_{j}^{S}\right\}$ vector. The iteration steps of the brute-force method look like a mapping from $\left\{X_{j}^{S}, Y_{j}^{S}\right\}$ space to $\left\{X_{j}^{S}, Y_{j}^{S}\right\}$ space, constructed by two kinds of least-squares fits. In this map view, $\left\{X_{j}^{S}, Y_{j}^{S}\right\}$, which is obtained as the limit of the iteration loops, is the fixed point of the map between $\left\{X_{j}^{S}, Y_{j}^{S}\right\}$ spaces. Thus, the solution of minimizing the residual error sum could be obtained by solving the fixed-point problem of this map. In order to solve the fixed-point problem, we apply a multidimensional Newton-Raphson method with a numerical derivative and SVD. Typically, this Newton-Raphson method has converged within 10 iterations and 10 seconds. Although this Newton-Raphson method converges very fast, its convergence sometimes fails when the measurement is not sufficiently accurate.

In order to establish both speed and robustness of the convergence, many kinds of fitting methods are implemented in our fitting code, and the driver codes switch the fitting methods with each other, corresponding to the converging state. In the usual case, the Newton-Raphson method is at first applied. If the convergence of the Newton-Raphson method fails, the CGPR method is used as a backup scheme. In the extreme case that the CGPR method fails to converge, the brute-force method is eventually used; however, the case of applying the brute-force method is very exceptional. Usually, we redo the measurement if the fitting fails.

Although both the beta function and the betatron phase at the BPMs are obtained from Eq. (5), the obtained results have three kind of ambiguities. One is $2 \pi n(n \in \mathbf{Z})$ arbitrariness of the betatron phase advance between BPMs, because the inverse trigonometric functions are multivalued functions. The second is the origin of the betatron phase advance, because Eq. (2) does not depend on the absolute phase. Third is normalization of the beta function, because Eq. (2) is not broken by replacing both $\beta_{i}^{M}$ and $\beta_{j}^{S}$ with $\lambda \beta_{i}^{M}$ and $\lambda^{-1} \beta_{j}^{S}$, respectively.

For $2 \pi n$ arbitrariness, we can apply the assumption that the betatron phase advance between neighboring BPMs is less than $\pi$ radians, because the density of the KEKB BPMs is high enough. The typical horizontal and vertical operation tunes of the KEKB rings are $(45.505,43.534)$ for LER and $(44.509,41.565)$ for HER. Thus, the average phase advance between neighboring BPMs is about $0.1 \pi$ radian.

For determining both the origin of the betatron phase and the scale of the beta function, we assume that the measured optics functions are close enough to the model. Although this assumption is not valid before the beta correction, the normalization using this assumption is convenient for visualizing the difference between the model and the measured optics functions. Regarding the arbitrariness of the phase origin, the origin of the measured betatron phase is chosen so as to minimize the sum of squares of betatron phase difference between the measurement and the model. As the result of the minimizing, the origin of the measured betatron phase is obtained by canceling the sum of the betatron phase difference between the measurement and the model:

$$
\sum_{i}\left[\phi_{\text {meas }}\left(s_{i}\right)-\phi_{\text {model }}\left(s_{i}\right)\right]=0 .
$$

For the scaling arbitrariness of the beta function, we assume that the expected value of the betatron phase advance $(<1 / \beta\rangle)$ is equivalent between the measurement and the model. Therefore, the scale of the measured beta function is adjusted to keep the inverse sum of the beta function at the BPMs between the measurement and the model:

$$
\sum_{i}\left(\frac{1}{\beta_{\text {meas }}\left(s_{i}\right)}\right)=\sum_{i}\left(\frac{1}{\beta_{\text {model }}\left(s_{i}\right)}\right)
$$

These three ambiguities are caused by the degree of freedom of Eq. (2). However, the direction of the betatron phase advance is already fixed by selecting the sign of $S_{i j}$ in Eq. (5). In the fitting of Eqs. (3) and (4), the result with an inverted sign against the assumption of the definition of $S_{i j}$ is unphysical. In such a case, either a change of 
the fitting method or the initial condition is necessary. According to our experiences, it is often caused by the error of the betatron tune measurement or the orbit drift. If the fitting fails in actual operation, we discard the data and redo the measurement.

\section{B. Correction scheme by using scale fudge factor of the quadrupole field}

In the beta correction scheme, we assume that the measured distortion of the optics is caused by an error in the field gradient of the quadrupole fields, and we try to correct the optics distortion by tuning the quadrupole field using fudge factors of the power supplies of the quadrupole magnets. In the magnet control system of the KEKB rings, the integration of the magnetic field strength for the $2(n+$ 1)-pole divided by the magnetic rigidity is defined by

$$
\frac{B^{(n)} L}{B \rho}=a_{f} K_{n}+\frac{b_{f}}{B \rho},
$$

where $a_{f}$ and $b_{f}$ are correction coefficients. We call these parameters fudge factors in this paper, and the design values of $a_{f}$ and $b_{f}$ are 1 and 00 , respectively. $L$ and $B \rho$ are the effective length of the magnet and the magnetic rigidity of the beam, respectively. $K_{n}$ is the normalized strength of the integrated magnetic field, defined by $\tilde{B}^{(n)} L / B \rho$, where $\tilde{B}^{(n)}$ is the $2(n+1)$-pole magnetic field component of the model without machine errors. We try to reproduce optics errors by introducing $a_{f}$ for the power supply of the quadrupole magnet family.

From first-order perturbation theory, the deviation of the beta function due to field gradient errors is described as

$$
\begin{aligned}
\frac{\triangle \beta_{\chi}\left(s_{i}\right)}{\beta_{\chi}\left(s_{i}\right)}= & -\frac{1}{2 \sin 2 \pi \nu_{\chi}} \triangle K_{\chi}^{j} \beta_{\chi}\left(s_{j}\right) \\
& \times \cos \left[2\left|\phi_{\chi}\left(s_{i}\right)-\phi_{\chi}\left(s_{j}\right)\right|-2 \pi \nu_{\chi}\right],
\end{aligned}
$$

where $s_{i}, s_{j}$, and $\triangle K_{\chi}^{j}$ are the location of the $i$ th BPM, the location of the $j$ th quadrupole magnet, and the error of the integrated field gradient of the $j$ th quadrupole magnet in the $\chi$ plane, respectively. In terms of the integrated quadrupole field strength $\left(K_{1} \equiv \frac{1}{B \rho} \int \frac{\partial B_{y}}{\partial x} d s\right), \triangle K_{x}^{j}$ and $\triangle K_{y}^{j}$ are described as $\triangle K_{1}^{j}$ and $-\triangle K_{1}^{j}$, respectively. The modulation of the betatron phase and the tune shift are given by following integral forms:

$$
\begin{aligned}
\triangle \phi_{\chi}\left(s_{i}\right)= & \int_{0}^{s_{i}}\left(\frac{1}{\beta_{\chi}\left(s^{\prime}\right)+\triangle \beta_{\chi}\left(s^{\prime}\right)}-\frac{1}{\beta_{\chi}\left(s^{\prime}\right)}\right) d s^{\prime} \\
= & \frac{1}{2 \sin 2 \pi \nu_{\chi}} \triangle K_{\chi}^{j} \beta_{\chi}\left(s_{j}\right) \\
& \times\left\{2 \sin 2 \pi \nu_{\chi} \sin ^{2} \min \left[\phi_{\chi}\left(s_{i}\right)-\phi_{\chi}\left(s_{j}\right), 0\right]\right. \\
& \left.+\sin \phi_{\chi}\left(s_{i}\right) \cos \left[2 \phi_{\chi}\left(s_{j}\right)-\phi_{\chi}\left(s_{i}\right)-2 \pi \nu_{\chi}\right]\right\},
\end{aligned}
$$

$$
\triangle \nu_{\chi}=\frac{1}{2 \pi}[\triangle \phi(C)-\triangle \phi(0)]=\frac{1}{4 \pi} \triangle K_{\chi}^{j} \beta_{\chi}\left(s_{j}\right) .
$$

In the case using the quadrupole magnet family as a corrector, Eqs. (10)-(12) are replaced by the sum over the individual quadrupole magnets of the quadrupole magnet family. In the following section, we deal as if all quadrupoles have individual power supplies; however, the result is easily extended to the case with a family of magnets driven by a single power supply.

The field gradient error can be written by

$$
\triangle K_{1}^{i}=K_{1}^{i} \triangle a_{f}^{i}, \quad \triangle a_{f}^{i} \equiv a_{f}^{i}-1,
$$

where $K_{1}^{i}$ is the design value of the $i$ th quadrupole magnet.

Substituting Eq. (13) into Eqs. (10)-(12), the relationship between the fudge factor of the $i$ th quadrupole family $\left(\triangle a_{f}^{i}\right.$ ) and the optical functions $\left[\triangle \beta\left(s_{i}\right) / \beta\left(s_{i}\right), \triangle \phi\left(s_{i}\right)\right.$, and $\triangle \nu]$ is obtained. In order to determine the fudge factor, we minimize the sum of squares of residual errors calculated by

$$
\begin{aligned}
e^{2} \equiv & \left(\frac{\pi}{\sin 2 \pi \nu}\right)^{2}\left|\triangle \nu-\left(\nu^{\text {meas }}-\nu^{\text {model }}\right)\right|^{2} \\
& +\sum_{i}\left(\left|\frac{\triangle \beta\left(s_{i}\right)}{\beta\left(s_{i}\right)}-\frac{\beta^{\text {meas }}\left(s_{i}\right)-\beta^{\text {model }}\left(s_{i}\right)}{\beta^{\text {model }}\left(s_{i}\right)}\right|^{2}\right. \\
& \left.+\left|\triangle \phi\left(s_{i}\right)-\left[\phi^{\text {meas }}\left(s_{i}\right)-\phi^{\text {model }} l\left(s_{i}\right)\right]\right|^{2}\right),
\end{aligned}
$$

where $\Delta \nu, \triangle \beta\left(s_{i}\right)$, and $\Delta \phi\left(s_{i}\right)$ are the response of the optical functions given by Eqs. (10)-(12) and the sum $\sum_{i}$ means the sum over all of the measured BPMs. The fudge factor $\left(\triangle a_{f}^{i}\right)$ is obtained by solving the linear equations of Eqs. (10)-(13) with SVD. The obtained fudge factor shows the correction factor to adjust the model optics so as to reproduce the measured one. Thus, the optics error is compensated by an iterating procedure for the fudge factor of the quadrupole magnet for each power supply using following scheme:

$$
a_{f \text { new }}^{i}=\frac{a_{f \text { old }}^{i}}{1+\triangle a_{f}^{i}} .
$$

In Eq. (14), equal weighting is applied to the individual square terms of the residual error. If the variance of the measured value is known, the individual square term in Eq. (14) should be multiplied by the inverse square of the standard deviation. In actual use, the extra weighting factor is applied in order to preferentially correct a specific optics parameter (for example betatron tune, beta function at interaction region).

In the beta measurement described in the previous section, we must pay attention to the measurement uncertainty. This measurement method cannot determine both the scaling factor of the beta function and the origin of the phase advance at the same time, because Eq. (2) is con- 
served by either a change of the origin of the phase or the scale of the beta function, while keeping $\beta_{i}^{M} \beta_{j}^{S}$ unchanged. If the optics distortion is sufficiently small, such an uncertainty is canceled out by normalization using the model optics. If the optics distortion is not sufficiently small, this uncertainty problem could be avoided by either introducing a fudge factor for the beta amplitude and the phase origin to Eq. (2) as the unknown quantity, or by constructing a residual error from the relative value between the neighborhood BPMs.

\section{Measurement and correction at the KEKB rings}

At KEKB, the beta measurement and correction method described in the previous sections has been implemented on SAD [10]. The SAD is a computer code complex for accelerator physics, developed at KEK since 1986. In its development, we have started to build a computer program complex for accelerator design work and beam simulations. In order to use the SAD for the online model optics of the accelerator commissioning, a general-purpose script interpreter, EPICS [11] channel access interface, and a GUI toolkit based on TCL/TK [12] are implemented into the SAD. Using an automation tool written in the script language on the SAD environment, we correct the ring optics after the routine maintenance conducted every two weeks.

In measurements at the KEKB rings, we use 6 steering dipole magnets to measure the horizontal beta function and 6 steering dipole magnets for the vertical beta function measurement. In order to avoid similar varieties of the measured closed orbit, the betatron phases of the steering dipole magnets are spread out as much as possible. We exclude the horizontal dipole kickers in the dispersive section in order to avoid any change of the circumference.

KEKB has a $2.5 \pi$ cell structure with noninterleaved sextupoles in the arc cells shown in Fig. 1. Two noninterleaved pairs of sextupole magnets are placed in a cell. In order to cancel out the nonlinear effect of the sextupole pair, a 4 by 4 transfer matrix between the sextupole pair is adjusted to $-\boldsymbol{I}^{\prime}$. $-\boldsymbol{I}^{\prime}$ is a 4 by 4 matrix similar to a negative identical 4 by 4 transformation, as follows:

$$
-\boldsymbol{I}^{\prime}=\left(\begin{array}{cccc}
-1 & 0 & 0 & 0 \\
C_{12} & -1 & 0 & 0 \\
0 & 0 & -1 & 0 \\
0 & 0 & C_{34} & -1
\end{array}\right)
$$

where $C_{12}$ and $C_{34}$ are arbitrary numbers. By using the $-\boldsymbol{I}^{\prime}$ transformation, the nonlinear kick of the sextupole generated by the closed-orbit distortion propagated from outside of the sextupole pair is cured by the paired sextupole. Therefore, we cannot use the steering dipole magnet within the sextupole pair to induce a closed-orbit distortion, because the single dipole kick within the sextupole pair makes the tune shift and the optics distortion, such as beta beat, $x-y$ coupling, and dispersion.

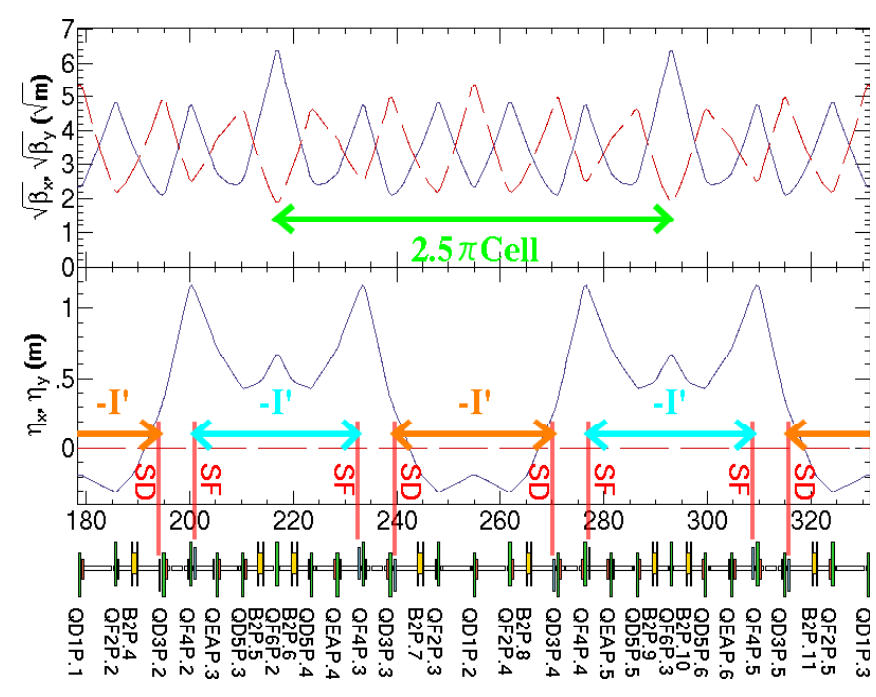

FIG. 1. (Color) Two periods of the $2.5 \pi$ arc cell of LER with beta and dispersion functions. In one arc cell, two $-\boldsymbol{I}^{\prime}$ sections are indicated by cyan and orange arrows. In this plot, the blue solid line and the red dashed line correspond to the horizontal and vertical optics functions, respectively.

To make the amplitude of the response of the closed orbit the same, the kick angle is scaled by the square root of the beta function at the steering dipole magnet. The typical kick angle for the orbit response measurement is about $40 \mu \mathrm{rad}$ for $30 \mathrm{~m}$ of the beta function. The typical peak-topeak amplitude of the kicked closed-orbit at the arc section is about $\pm 0.5 \mathrm{~mm}$. The Newton-Raphson method in the beta function fitting has normally converged until 6 iterations. The typical rms value of the residual error of the orbit response fitting is within $2 \mu \mathrm{m}$, except for the vertical measurement of HER. The typical rms value of the vertical fitting residual at HER is about $4 \mu \mathrm{m}$.

In the beta correction, the quadrupoles used to make the $-\boldsymbol{I}^{\prime}$ condition in the arc and the quadrupole at the injection point are excluded from the corrector candidates. In the usual correction, we apply 10 times extra weightings for the tune errors in Eq. (14), and 0.05 is used as the relative tolerance for truncating the small singular values of the response matrix. The typical time period of both the measurement and the correction of the beta function is $7 \mathrm{mi}-$ nutes. In the usual case, such as a correction after regular maintenance, the beta correction has normally converged within 3 iterations. After corrections, the typical residual error of the beta function, $\triangle \beta / \beta$, is about $5 \%$ in rms and the betatron tune is corrected into $\pm 5 \times 10^{-4}$. In the KEKB rings, the $a_{f}$ fudges of the quadrupoles are almost distributed within $\pm 5 \times 10^{-3}$. The typical range of the change of the $a_{f}$ fudge factor in the on-momentum correction procedure is $\pm 2 \times 10^{-4}$.

As another corrector, we use cosinelike bump orbits at the sextupole pairs. By making the displacements of the horizontal orbit at the sextupole pair with the same sign, the sextupole pair is effectively worked as a quadrupole 


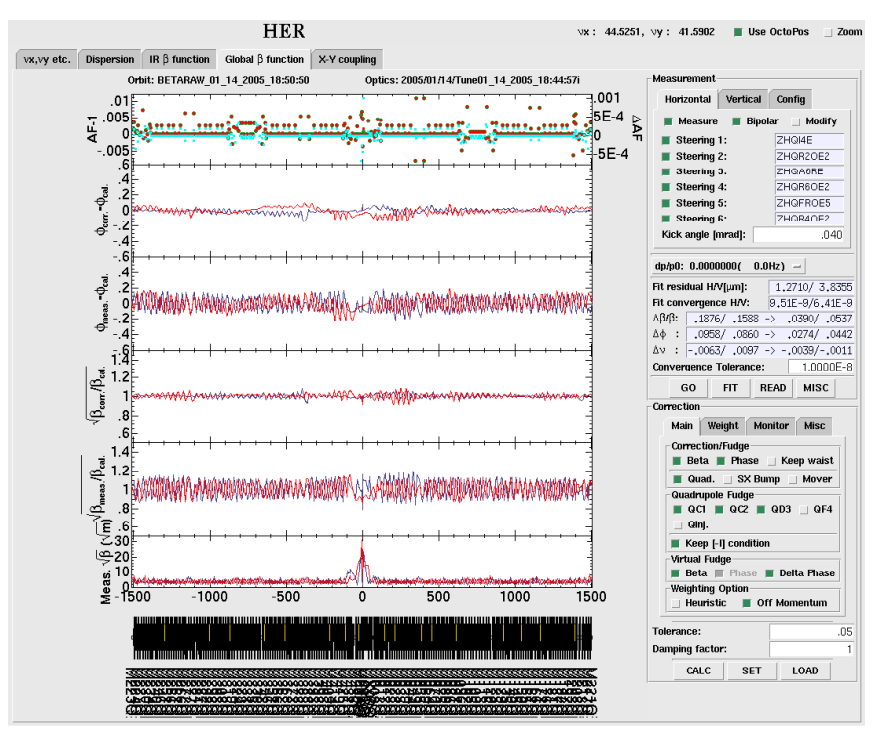

(a)Measurement before Correction

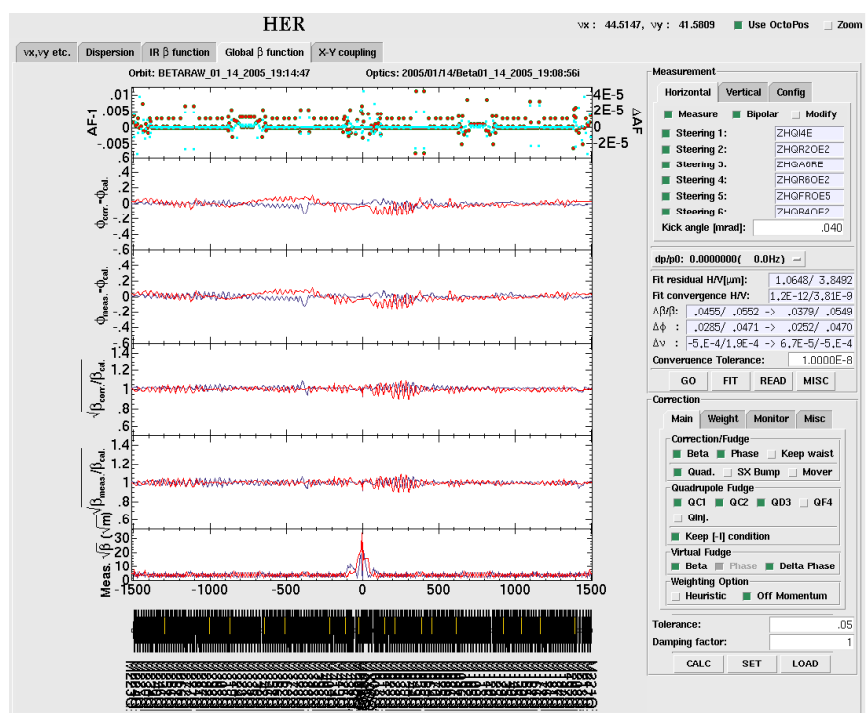

(b)Measurement after the second

FIG. 2. (Color) Results of the beta measurement before (a) and after (b) the correction. In the first row of the graphs, the current and desired values of $a_{f}$ are shown by green and red points, respectively. The measured value of the phase advance error and the squareroot ratio of the beta function are shown in the $3 \mathrm{rd}$ and the 5 th rows, respectively. The 2 nd and 4 th rows show a prediction after the correction. In these graphs, the blue and red lines correspond to the horizontal and vertical optics functions, respectively.

without producing any horizontal dispersion. These sextupole bumps are used to correct the beta function distortion localized in the arc section.

Figure 2 shows an example of the beta measurement before and after the correction. At a measurement before correction, the relative error of the beta function $(\triangle \beta / \beta)$ exceeds $15 \%$ in rms, and a betatron tune shift larger than $5 \times 10^{-3}$ is observed. At the measurement after two iterations of the beta corrections, the relative error of the beta function becomes less than $5 \%$ in rms and the betatron tune shift is corrected into $5 \times 10^{-4}$.

\section{OFF-MOMENTUM BETA MEASUREMENT AND CORRECTION}

\section{A. Off-momentum beta measurement}

In order to measure the beta function with the momentum deviation, $\triangle p / p_{0}$, we shift the momentum of the beam by changing the $\mathrm{rf}$ frequency of the accelerating cavity. The relationship between the momentum and the rf frequency is given by

$$
\frac{\triangle f}{f_{0}}=-\left(\alpha_{c}-\frac{1}{\gamma^{2}}\right) \frac{\triangle p}{p_{0}},
$$

where $f, \alpha_{c}$, and $\gamma$ are the $\mathrm{rf}$ frequency of the accelerating cavity, the momentum compaction factor, and the Lorentz factor, respectively. The slippage factor $\left(\alpha_{c}-\gamma^{-2}\right)$ of the KEKB ring is about $3 \times 10^{-4}$, and the nominal frequency of the rf cavity is $508.886 \mathrm{MHz}$. In the examples shown in the next subsection, the beta measurement was performed with five different frequency shifts: $-400,-200, \pm 0$, +200 , and $+400 \mathrm{~Hz}$. The range of $\triangle p / p_{0}$ is $\pm 2 \times 10^{-3}$, which corresponds to these frequencies.

\section{B. Correction scheme using an amplitude fudge factor of the sextupole field}

KEKB has 54 independent families of sextupole magnets in LER and 52 families in HER, respectively. We assume that a deviation of the sextupole field is the source of off-momentum optics distortion. Based on this assumption, the off-momentum correction becomes a problem of how to find a parameter set of the sextupole strength so as to minimize the residual error between the model optics and the measured optics at the measured momenta. This residual error can be described in terms of the measured optical functions: betatron tunes, beta functions, and betatron phase advances.

An off-momentum optics correction should be performed by adjusting the $a_{f}$ fudge factor of the sextupole power supply. The change in the sextupole field strength $\left(K_{2} \equiv \frac{1}{B \rho} \int \frac{\partial^{2} B_{y}}{\partial x^{2}} d s\right)$ affects both the off-momentum and the on-momentum optics, because it generates the normal and skew quadrupole fields, depending on the horizontal and vertical displacement of the closed-orbit at the sextupole magnet. In fact, we use this off-center orbit effect for correcting the on-momentum $x-y$ coupling, dispersions, and beta functions in the horizontal and vertical planes [2]. Thus, it is preferred to solve the $K_{2}$ correction fudge of the off-momentum correction simultaneously with the on- 
momentum correction. However, it is difficult to obtain an effective correction fudge from such simultaneous equations. For solving the off-momentum correction with the on-momentum correction, the absolute value of the orbit displacement at the sextupole magnet is required, and the size of the matrix of the simultaneous equations is enlarged compared to the matrices without the on-momentum correction.

To simplify the correction scheme, we performed the correction only for the chromatic term of the optical functions proportional to $\triangle p / p_{0}$, and ignored the orbit offset at the sextupoles. In order to compensate for the side effect by changing the sextupole field, both on-momentum and offmomentum corrections should be iterated alternatively.

One of the simplest error functions for the offmomentum correction should be written as a quadratic form by the relative change of the sextupole field, $\triangle K_{2} / K_{2}$. This function is obtained from the quadratic form of the errors of the optical functions as well as the response of these optical functions to the change of the sextupole field.

The error function between the measured optics and the model is given by

$$
\begin{aligned}
e^{2}= & \sum_{k}\left[(2 \pi)^{2}\left(\Delta \nu_{\text {meas }}^{k}-\Delta \nu_{\text {model }}^{k}\right)^{2}\right. \\
& +\sum_{j}\left[\Delta \phi_{\text {meas }}^{k}\left(s_{j}\right)-\triangle \phi_{\text {model }}^{k}\left(s_{j}\right)\right]^{2} \\
& \left.+\sum_{j}\left[\triangle \hat{\beta}_{\text {meas }}^{k}\left(s_{j}\right)-\triangle \hat{\beta}_{\text {model }}^{k}\left(s_{j}\right)\right]^{2}\right]
\end{aligned}
$$

where $s_{j}$ is the location of the BPMs and $k$ means that the optical functions correspond to the momentum of the $k$ th off-momentum measurement. The momentum-dependent terms of the optical functions are defined as follows:

$$
\begin{gathered}
\triangle \nu^{k}=\nu^{k}-\nu^{0}, \\
\triangle \phi^{k}(s)=\phi^{k}(s)-\phi^{0}(s), \\
\triangle \hat{\beta}^{k}(s)=\frac{\beta^{k}(s)}{\beta^{0}(s)}-1,
\end{gathered}
$$

where ${ }^{0}$ means the optical function on the reference momentum. Using the model optics of SAD, the response matrix of the model optics is easily obtained by numerically differentiating the optical functions: $\Delta \nu_{\text {model }}$, $\triangle \beta_{\text {model }}(s)$, and $\triangle \phi_{\text {model }}(s)$. Minimizing $e^{2}$ in Eq. (18) with a given response matrix is equivalent to solving the linear equations by SVD. A linear equations can be written as follows:

$$
\begin{aligned}
& \left(\begin{array}{c}
2 \pi\left(\Delta \nu_{\text {meas }}^{k}-\Delta \nu_{\text {model }}^{k}\right) \\
\triangle \boldsymbol{\phi}_{\text {meas }}^{k}\left(s_{j}\right)-\Delta \boldsymbol{\phi}_{\text {model }}^{k}\left(s_{j}\right) \\
\triangle \hat{\boldsymbol{\beta}}_{\text {meas }}^{k}\left(s_{j}\right)-\Delta \hat{\boldsymbol{\beta}}_{\text {model }}^{k}\left(s_{j}\right)
\end{array}\right) \\
& =\left(\begin{array}{ccc}
2 \pi \boldsymbol{I} & \\
& \boldsymbol{I} & \\
& \boldsymbol{I}
\end{array}\right) \boldsymbol{M}_{s_{j}}^{k} \boldsymbol{K}_{2}\left(\triangle \boldsymbol{a}_{\boldsymbol{f}}\right),
\end{aligned}
$$

where $\boldsymbol{M}_{s_{j}}^{k}$ is a part of the response matrix for the $k$ th momentum and location $s_{j}$, and $\boldsymbol{K}_{2}$ is the diagonal matrix of the sextupole field strengths; $\triangle a_{f}$ is the fudge used to adjust the model optics to the measurement.

In order to reduce the error between the model optics and the real machine, the calculated sextupole fudge factor, $\triangle a_{f}$, is applied to the power supply of the sextupole magnet by the following scheme:

$$
a_{f \text { new }}=\frac{a_{f \text { old }}}{1+\triangle a_{f}}
$$

where $a_{f \text { old }}$ and $a_{f \text { new }}$ are the $a_{f}$ fudge factors of the power supply before and after a correction, respectively.

On the other hand, the sextupoles are optimized during daily machine tuning. We do not want to lose the stability of the machine operation by changing the actual strengths of the sextupole magnet. Thus, for keeping the excitation current of the sextupole magnet, we update the sextupole strength of the model by

$$
K_{2 \text { new }}=K_{2 \text { old }}\left(1+\triangle a_{f}\right),
$$

where $K_{2}$ old and $K_{2 \text { new }}$ are the sextupole strength before and after the correction, respectively. After updates using Eqs. (23) and (24), the off-momentum response of the model optics comes close to that of the actual machine.

\section{Measurement and correction at the KEKB rings}

Our off-momentum correction scheme was tested on LER of KEKB. The typical time to measure the offmomentum optics was about 50 minutes in total for 5 different momenta. The SVD tolerance for the correction fudge calculation was chosen to be 0.1 . The first measurement result of the distribution of the off-momentum beta function is shown in Fig. 3. By applying the correction to the model optics using Eq. (24), the blue dashed model line in Fig. 3 is modified to the green dotted line, and becomes close to the red solid line of the measured one. A calculation of correction fudge to the model optics was successfully performed.

Figures 4 and 5 show the trend of the distribution and the $\mathrm{L}^{2}$-norm of the correction fudge for the sextupole. These $\mathrm{L}^{2}$-norm, which would be correlated with the correctable residual error of the sextupole field, shows a decreasing tendency, except for a measurement on January 13, 2006. This exceptional point would come from a change of the machine condition during the shutdown, because KEKB 


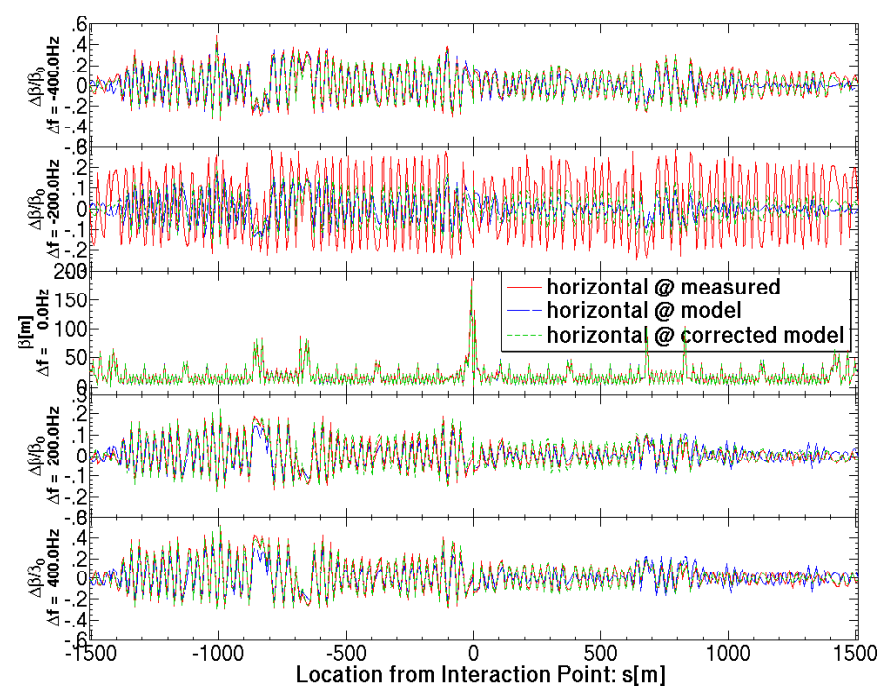

(a)Horizontal

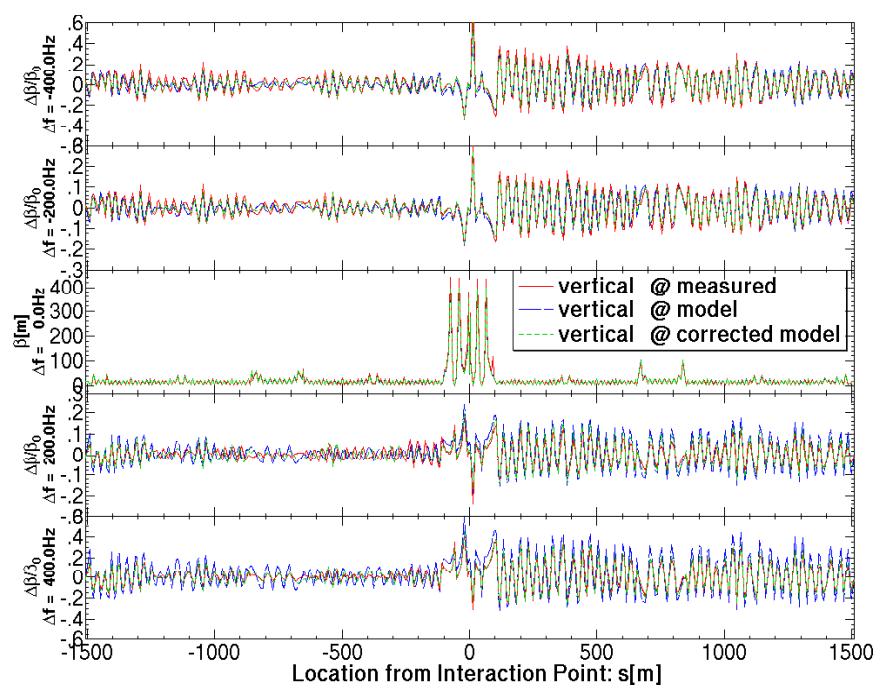

(b) Vertical

FIG. 3. (Color) Measured and model beta functions of the KEKB LER on November 12, 2005. The 3rd row shows the beta function, while the other rows show the chromatic deviations of the beta function. In these plots, red solid line, blue dashed line, and green dotted line correspond to the measurement, model before the correction, and model after the correction, respectively.

had a winter shutdown from December 26, 2005 to January 13. The correction series appears to be converging.

The off-momentum beta correction scheme has shown convergence. However, we have not yet obtained any obvious gain by the off-momentum correction on the machine performance. In order to make the correction more

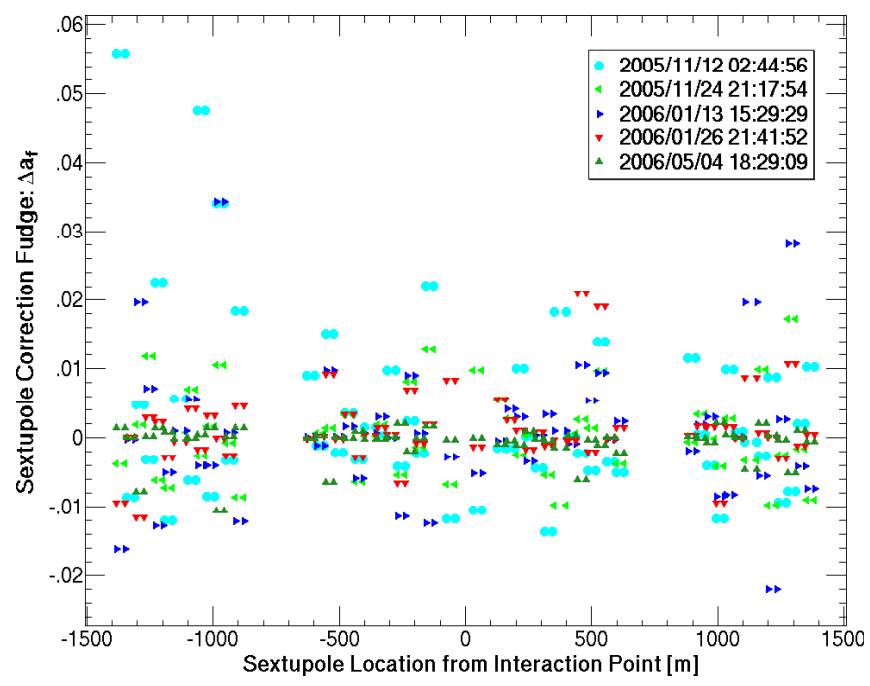

FIG. 4. (Color) Distribution of sextupole correction fudge $(\triangle$ $a_{f}$ ) obtained from the measurement. The horizontal axis shows the position of the corrector sextupole described by the distance from the interaction point (IP) along the design orbit. The vertical axis shows the correction factor for the $a_{f}$ fudge of the sextupole power supply at the individual off-momentum corrections. The 5 kinds of symbols correspond to the offmomentum corrections performed at 5 different times. effective, we consider how to expand the momentum span of the measurement. Our regular optics correction is performed within one hour per ring. For introducing an offmomentum correction into the regular correction procedure, speed-up of the measurement is necessary. For example, if the betatron phase measurement of the whole ring were to be available by introducing the single-pass BPMs, the off-momentum optics measurement could be speeded up. As a feasible plan for the present measurement system,

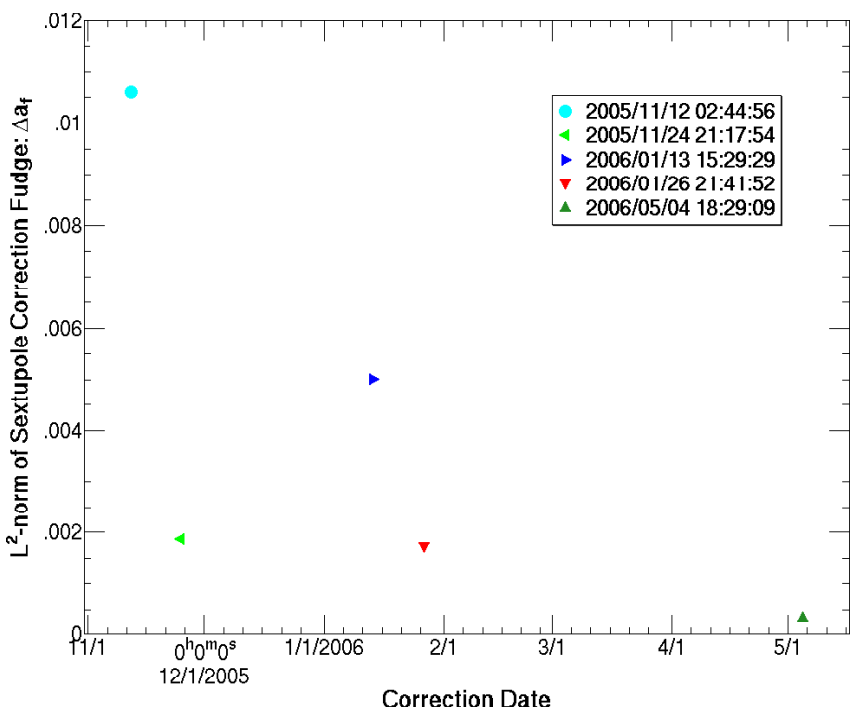

FIG. 5. (Color) Trend of the $\mathrm{L}^{2}$-norm of sextupole correction fudge, $\triangle a_{f}$. The horizontal axis shows when the off-momentum correction is applied. The vertical axis shows the $\mathrm{L}^{2}$-norm of the $\triangle a_{f}$ distribution in Fig. 4. 
we investigate the speed-up by reducing the number of the single kicks to measure. Although this may sacrifice the reliability, the measurement time would be reduced by almost half at the maximum.

\section{CONCLUSION}

The on-momentum beta measurement and correction work well as a part of the optics correction of the regular operation of KEKB. It has been very helpful operating the KEKB rings near to half-integer resonance lines.

\section{ACKNOWLEDGMENTS}

The authors acknowledge N. Akasaka for developing the optics correction at the early stage of KEKB. We also thank M. Masuzawa, M. Tejima, and S. Hiramatsu for developing BPM, beam-based alignment, and BPM calibration by beam, and also Y. Funakoshi, and all of the KEKB accelerator group, for giving us the chance to develop the scheme.

[1] KEKB B-Factory Design Report, KEK Report 95-7, 1995, http://www-acc.kek.jp/kekb/publication/KEKB_design_ report/KEKB\%20Design\%20Report.html.

[2] KEKB Accelerator Papers, NIM Section A (NorthHolland, Elsevier, 2003), Vol. 499, Issue 1, pp. 1-233.
[3] A. Morita, H. Koiso, Y. Ohnishi, and K. Oide, in Proceedings of the 2005 Particle Accelerator Conference, Knoxville, Tennessee, USA, 2005, p. 802.

[4] P. Castro, J. Borer, A. Burns, G. Morpurgo, and R. Schmidt, in Proceedings of the 1993 Particle Accelerator Conference, Washington, DC, USA, 1993, p. 2103.

[5] M. Yoon, H. K. Jeong, and T. Lee, in Proceedings of the 1996 European Particle Accelerator Conference, Gran Sitges Hotel, Sitges, Barcelona, 1996, p. 1420.

[6] H. Harrison and S. Peggs, in Proceedings of the 1987 Particle Accelerator Conference, Washington, DC, USA, 1987, p. 1105.

[7] G. H. Hoffstaetter, J. Keil, and A. Xiao, in Proceedings of the 2002 European Particle Accelerator Conference, Paris, 2002, p. 407.

[8] E. D. Courant and H. S. Snyder, Ann. Phys. (N.Y.) 3, 1 (1958).

[9] W.H. Press, S. A. Teukolsky, W.T. Vetterling, and B.P. Flannery, Numerical Recipes in C: The art of Scientific Computing Second Edition (Cambridge University Press, Cambridge, England, 1993), pp. 420-425.

[10] Strategic Accelerator Design, http://acc-physics.kek.jp/ $\mathrm{SAD} /$.

[11] Experimental Physics and Industrial Control System, http://www.aps.anl.gov/epics/.

[12] Tool Command Language and Tk GUI toolkit, http:// www.tcl.tk/software/tcltk/. 